\title{
Symbol Consumption in New Social Media -- Analysis of Chinese Memes
}

\begin{abstract}
Yuqiao $\mathrm{Liu}^{1, *}$
${ }^{1}$ City University of Hongkong, Hongkong, China

*Corresponding author. Email: yuqiaoliu2-c@my.cityu.edu.hk

ABSTRACT

As the new generation of Chinese youth subculture that is driven by the new media of the Internet, the values of visual consumerism have become morediversified, Evans and Stuart Hall (1999) state that "the mechanically and electronically reproduced image is the semantic and technical unit of the modern mass media and at the heart of post-war popular culture"i[1]. This perdition holds much truth today and the 'meme' is the best representative production, furthermore, meme's cutting, fixing, magnifying, and recombining objects decentralize political power as never before. Memes have become one of the languages of social media, particularly on WeChat, Weibo and Tieba China's popular social media apps and platforms. Memes are a combination of text and images or pure images, and they have become a means for youth to express their emotions and collective revelry. Meme culture has changed the traditional art and aesthetic impact and to the ways in which people consume images. In this era of high meme consumption, the centralization of media has been weakened by image consumption and transformation. In a consumer society that is full of images, memes can also become a motivating factor for the consumption of consumer consumption and for increasing consumer loyalty to a brand.
\end{abstract}

Keywords: Visual culture, image consumption, photographic visualization, Patriotis

\section{INTRODUCTION}

Due to the rapid development of the social economy, the material level of people continues to improve. According to Maslow's hierarchy of needs, the demands of contemporary youth have changed from the social attribute of basic physiological needs to that of mental spiritual needs. In modern Chinese society, people have become intoxicated with the sensory pleasure various forms of mass media provide, and their critical thinking abilities have been degraded as a result. Image entertainment has now become an ideology, and people praise the cultural concept of "amusing ourselves to death" through sensory stimulation and a lack of rational thinking. Image consumption is an incredibly representative phenomenon resulting from the development of images and the Internet. This reflects people's cultural lives and is also the primary means for investigating young people's ways of thinking. By establishing who uses and produces memes and the development trajectory of memes, we can better anticipate future graphics development and the use of marketing.

This paper is represented by meme consumption as the breakthrough point and analyzes the relationship between visual art and consumer behavior. It examines the historic consumption of images, the ways in which art can use characters to express emotions with facial expressions, and analyzes the transition of visual arts in the media era. Consumers have changed from worshiping the beauty of traditional images to using them for entertainment. Visual art has completed the transformation of its functions, and now anyone can participate in the production of image art. Memes are a typical representative of this.

\section{IMAGE CONSUMPTION DEVELOPMENT AND FUNCTION TRANSFORMATION}

\subsection{The eyeball economy under media development}

Since 1992 image theorist Michel declared that we were moving from the 'linguistic turn' to the 'cultural turn' to the 'pictorial turn, pictorial turn (Michel, 1995) ${ }^{i i}$ [2], eyeball economy and image analysis soon informed the realm of media and visual studies. With the rise of 
new technology and the development of mobile internet, producing, retrieving and sending image has become extremely convenient. Nowadays, in a massive information era, the human experience is more visual than ever before. People's attention has become a form of economic goods that is used to obtain economic benefits. Aided by the powerful modern media society, the eyeball economy is more active than ever. Image consumption generally requires no actual purchase, unlike buying a painting or buying an item as a result of advertising. A user glancing at an image can help internet products gain profits from other companies. Attention has become a tradable commodity and it is now the main feature of the eyeball economy.

According to Stuart Hall, in language, we use signs and symbols - whether they are sounds, written words, electronically produced images, musical notes, even objects -to stand for or represent to other people our concepts, ideas and feelings. Language is one of the 'media' through which thoughts, ideas and feelings are represented in a culture (1997, Hall) ${ }^{i i i}$ [3]. Meme as a kind of symbols, Memes have become symbols that are necessary for the daily social communication of the younger generation. They have become part of the language of the Internet era in China, particularly on the social media platform, WeChat. Memes convey certain emotions, concepts, and thoughts and can be spread rapidly.

The meme, as a new expression created by the youth, can appear in a variety of distinguishable forms. By using a multi-dimensional and visual combination of image, text and action (GIF) the visual and communicative element of the sender greatly improves. In his book Image Politics (DeLuca, 2005) iv [4], DeLuca repeatedly emphasizes with all cases that images have the irreplaceable power of social mobilization and discourse construction. In online communication, memes have a particular advantage in their ability to grab someone's attention, so more than words. Secondly, the collocation of images and texts is conducive to the construction of visual effect. In addition, a concise supporting caption can help netizens improve the visual understanding and cognition of discourse, helping author to express emotions and increasing its appeal. Third, familiar images and expressions of net figures or celebrities are used in the form of clippings and recreated in Photoshop, celebrity benefits have own communication effect. Memes are hold a narrative, which can enrich the polyphonic information of the meme.

\subsection{Media has changed the consumption of images}

Before the industrial age, images generally came from traditional art, such as sculpture or painting. Changes in the ways artists portray facial expressions also reflect the change in image function and consumption. The convenience of reproduction and secondary editing of pictures by media has accelerated the diversification of image consumption.

In the history of ancient image culture, the expression of people in a particular mood exaggerated expression has never been the focus of plastic arts, and would even be avoided. Laocoön and His Sons is one of the most famous sculptures in all of art history and has received praise over centuries for its complex facial detail. "However, this pain expresses itself without any sign of rage either in his face or in his posture. He does not raise his voice in a scream which Virgil describes his Laocoon as doing. Rather he emits the anxious and subdued sigh described by Sadolet (Lessing, 1766) v [5]."

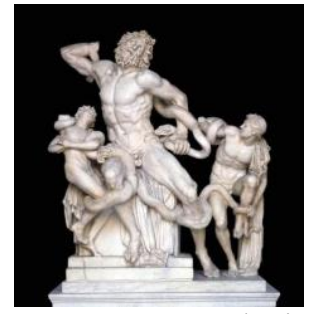

Figure1: Laocoön and His Sons

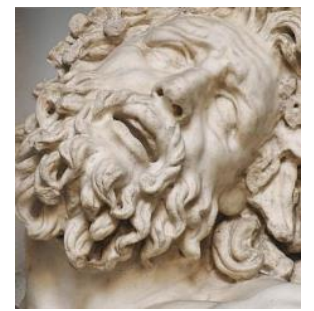

Figure2: Magnified expressions

\section{Figure1. Laocoon's head, Wikimedia Commons}

According to Lessing, unlike the effect of poetry, the visual artist must cover up all the excesses and mitigate the overflow of emotion, because to present a creation of beauty is an artist's duty not to depict something excessive painful. This is exactly the reason why the Trojan priest's mouth barely open. Only after the birth of the romantic period and the aesthetic reconstruction of artistic self-discipline were natural emotions expression properly accepted and depicted in modern art, and exaggerated and deformed facial expressions portrayed in visual art, just like the exaggerated facial expressions of so many memes.

However, contemporary facial expression in images is not influenced by modern painting art. Fundamentally, facial expression in memes is the invention of photography. Under certain emotional conditions, photography can effectively capture expressions. Second, creation like cutting, fixing, magnifying, and recombining objects make face and facial expression become the center of image culture as never before. All kinds of exaggerated facial expression photography, 
whether selfie or other photography, are an important node in the visual development of photography.

In modern world history, photographs have been doctored for political purposes. For example, to idealize someone by transforming them into a divine-like figure (Wu Hung, 1999)vi [6]. Remove other characters, visual focus can have a powerful effect and propel them into an unparalleled position in history, as was true during the Chinese revolution. During this period, portraits of prominent government leaders like Sun Zhongshan, Mao Zedong were bought and hung in homes, cars and under glass in desks.

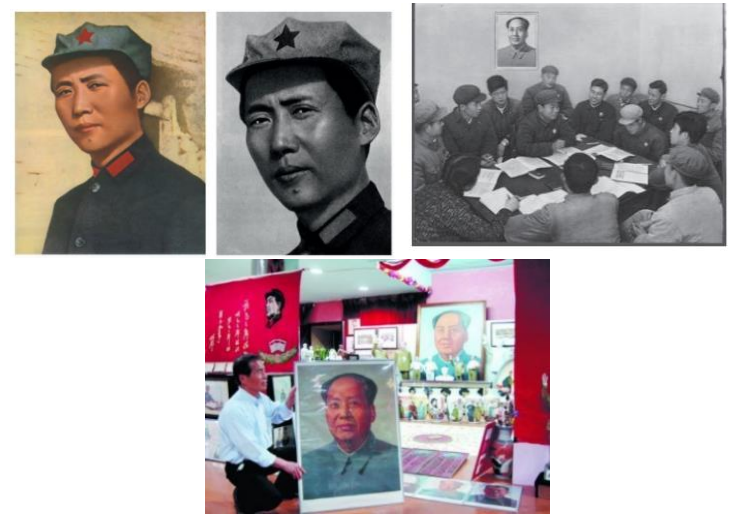

Figure3:Mao's political portrait used by the government and sold by artists.

In China, old political imagery is dominated by the deification of leading figures. For example, Mao's, portrait was hung in many Chinese homes between 1949 to 2000 . Some would even go as far as buying a pendant which embedded his portrait for exorcism and blessing. We begin to realize that the distortion of photographs does not always serve political propaganda. In fact, it is accepted and employed by society at large, since everyone is attracted by ideal, 'sublimated' images of themselves (Wu Hung 1999)vii [7].

\subsection{Self-expression and identity in the new image market}

In 1970, French sociologist Jean Baudrillard put forward the concept of "consumer society". The main forms of image consumption in the media era include magazines, advertisements, TV shopping, and online shopping. Image consumption is used at the first level to facilitate the creation of fantasies about products and scenarios by consumers as a means of achieving the purpose of desire consumption. At the second level, image consumption makes people construct their world views based on images. The consumption of images also represents the groups which consumers come from, represents their status, power, interests, identity and so on, consumers perceive matching of spiritual ideas.
Today, Young people in recent generations are highly sensitive to images, and they have a high capacity for creating, analyzing, and using images. In addition to being used for communication in daily life, it is also common for memes to take on social form of expression. By using memes as part of communication, thus netizens have felt a sense of empowerment and have begun to decentralize the discourse power of media. As the power of media content has shifted, young people use memes to emphasize their sense of participating and achieve the satisfaction of self-expression and self-construction through image interaction.

In the context of semiotics, any ideographic behavior is inseparable from the participation of symbols, and they are created to convey a certain meaning to others. Memes are symbols, and their consumption by young people reflects their same identity. Young people have a strong need for self-identity and group identity in the current "consumption society". By using the same kind of meme, groups of young people can create their own self-identity and find companions with similar beliefs or interests. For example, there is a significant difference between memes that are used by old people and those used by young people. Old people prefer colorful memes with large fonts and many of the characters are babies, old people, or landscapes, whereas young people have a preference for memes with exaggerated expressions, pay more attention to fun and the use of multiple scenes expression.

\section{ANALYSIS OF MEME CONSUMPTION BEHAVIOR FROM A CONSUMERISM PERSPECTIVE}

In Baudrillard's "consumer society", we are living in a world where consumption is the symbolic meaning, which is the true value of goods. People's image consumption is influenced by the shock of movies, the endorsement of celebrities, and the temptation of advertisements. Images arouse consumers' desire and passion. Image consumption is not only to satisfy needs, but also to pursue desires that are difficult to completely satisfy. The value of goods has lost its leading position in terms of consumption, and the symbolic meaning of the value of goods and services represents who they are and what they pursue. Commercialization has gradually spread into the symbol field and people now pay greater attention to the value of symbols. A meme is also a type of symbol and there is a complete meme production chain. A steady stream of memes infiltrates the current network space and are produced for people's consumption. With the support of technology and the abundant need, people's visual senses and emotional pursuit are released to a certain extent by the consumption of memes. 


\subsection{The main consumers of memes in China}

Memes were first active appeared on Tieba and QQ, Wechat. The character's face is magnified in the innermost layer. The majority of them are from internet stars and Internet Celebrities. The exaggerated expressions of Hong Kong film star Zhang Xueyou, basketball superstar Yao Ming, curator Jin and Xiaopang are the most popular. The outline of expression makes meme more accurate and vivid in expression compared with the text, and the emotions of the publisher can be felt visually at the first glance. People use the expressions rendered by the characters in the movies to perpetuate some of their current emotions. However, it can be the same or different from the emotion of the first shoot. For example, this picture of Choi Seong-guk was taken in a screenshot of movie Mr Kim vs Mr Kim vs Mr Kim. He was amused by a child, at that time, it was really sincere happiness, but when used in the meme, it turned into a mocking and speechless emotion.

Nowaday the emergence of memes in China has enriched the daily lives of social media users, while also updating the way of communicating among the younger generation. According to statistics relating to WeChat users. meme usage during the Spring Festival in 2017, a total of 16 billion memes were sent, including $37 \%$ that were sent by post-90s WeChat users. It can be seen that memes play a crucial role in communication between WeChat users. As the communication habits of WeChat users have changed, an increasing number of users choose to use memes for expressing their emotions, and some even communicate using memes without text, pictures, or audio. In the media-dominated communication environment of WeChat, users have gradually formed a new communication method and consumption experience through the use of memes.

\subsection{Meme characters}

Different to traditional art forms, people have a willingness to consume beautiful characters, particularly images of beautiful women and beautiful men under the male gaze. As the modern meme has developed, people have formed a stronger definition and inclusiveness of beauty, and the more exaggerated and dramatic memes become, the more effectively they can be spread.

But people's photographs are still the main source of memes, with netizens using the faces of popular culture figures including internet celebrities, Olympic athletes, and entertainment stars as the main subject of the memes. Wells (2011) described the first capture of reality as the after-image, and as the reader of the photo, we see differently than what the photographer originally intended, a second life of the image. This implies the decoding of the image is separate from the encoding of the image (Evans \& Hall, 1999; Hall, 1997; Newton, 2001; Smith et al., 2005) viii [8]. That is, everyone that views a photo brings along his or her personal biases, opinions, and philosophies that may influence their reception of and perception of the meaning of the photograph. Young people use collage, image choice and recombination to create use memes to express sentiments toward political issues, identities and virtual self-revelry. These memes reveal his composition expression. The character's face is magnified in the innermost layer. The majority of them are from internet stars and Internet Celebrities. The exaggerated expressions of Hong Kong film star Zhang Xueyou, basketball superstar Yao Ming, curator Jin and Xiaopang were an early favorite. Due to the ease of using tools to create memes, anyone can now customize memes according to their favorite roles, and Internet celebrities have become the mainstream roles in memes.

The outline of expression makes meme more accurate and vivid in expression compared with the text, and the emotions of the publisher can be felt visually at the first glance. Because each photograph is only a fragment, its moral and emotional weight depends on where it is inserted. A photograph changes according to the context in which it is seen. Each of these situations suggests a different use for the photographs but none can secure their meaning (Susan, 1973). People use the expressions rendered by the characters in the movies to perpetuate some of their current emotions. However, it can be the same or different from the emotion of the first shoot. For example, this picture of Choi Seong-guk was taken in a screenshot of movie Mr Kim vs Mr Kim vs Mr Kim. He was amused by a child, at that time, it was really sincere happiness, but when used in the meme, it turned into a mocking and speechless emotion.

\subsection{Meme civilian artist}

The core of image culture during the Internet era is how people are not simply the recipients of culture, but also produce it. Netizens can produce their own cultural pleasure through the creation of humorous, exaggerated, and bold memes. There are currently two main creation and consumption sources for memes. One is created by professionals or teams via an online meme shopping platform. WeChat users spend money and choose memes based on their preferences and habits and then download them. Favorites include "cute little brother", popular stars from TV series, and popular celebrities. The revenue from selling memes through the platform is beyond imagination. In 2017, an university student who came from Hangzhou, sold her meme on WeChat. She used only two hours to draw but this meme favorited by most young people and helped her earn more than $160000 \mathrm{rmb}$.

At the same time, WeChat users can create memes based on their imaginations by using one of many meme creation apps. The objects of creation can include relatives, friends, users themselves, or their favorite 
stars. The App can automatically recognize faces through the photo and synthesize them into meme template, within five minutes, everyone can come up with a hilarious design

During my investigation, I discovered that there are various explanations for memes' becoming a social tool and it popularity and the reason why users are willing to spend.

\section{THE BRAND MARKETING POWER OF MEMES}

With the change in media consumption, many consumers tend to skip ads they don't want to see, so humor and thoughtful media marketing can appeal more to consumers in this era. For professional meme marketers, memes are a low-cost means of quickly responding to hot news, establishing connections with fans, and enhancing brand impressions.

For example, Nike used Kobe's retirement as an opportunity to launch a series of animated comic memes on Weibo that received more than three million views and 100,000 retweets, creating what was arguably the most successful marketing campaign of all time.

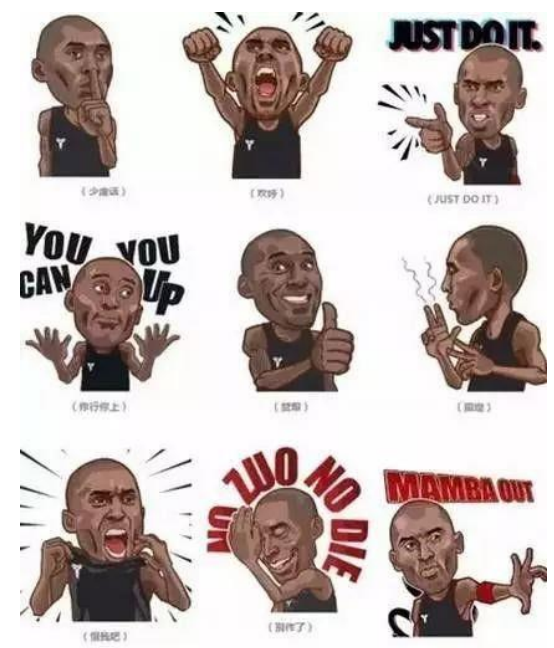

Figure4: Kobe's memes created by Nike

Another successful marketing campaign that used a celebrity meme was Mengniu (one of the top three milk companies in China), who launched a series of "I am Messi" memes during the 2018 World Cup on Weibo. Of these, the "Born strong" was seen over 7.12 billion times on social media. Although Mengniu spent more than 100 million dollars in sponsorship fees, the company successfully used meme marketing to gain a higher exposure. As a national children's drink, Wangzai milk reinvented its IP (intellectual property) image, created a set of IP memes for all 56 ethnic groups in China, and used them in the production design for the 70th anniversary of the founding of the People's Republic of China. The patriotic love stimulated by these memes led to loyal fans wanting to collect a complete set, which greatly stimulated consumption.

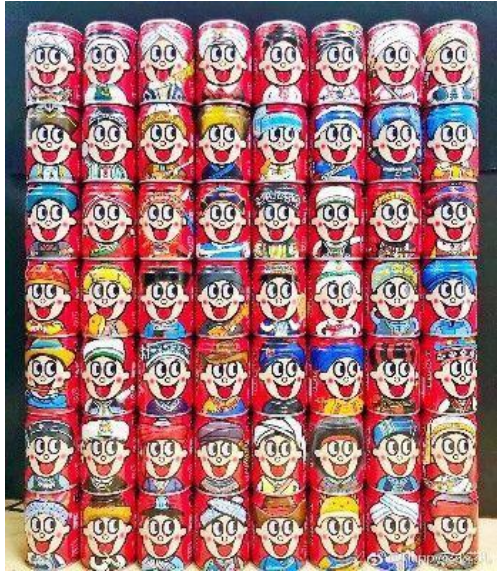

Figure5:Wangzai milk's IP memes for all 56 ethnic groups in China

Secondly, an increasing number of marketers are using memes to establish online brand communities, connect with fan bases, and enhance brand impressions. For example, Game For Peace (a mobile game created by Tencent) cooperated with Xiao Lan (a meme character on WeChat), leading to players having more fun during game communication. Lifuquan has collaborated with XiaoZaizi for the promotion of a new product, using popular memes on WeChat to offer users free downloads from the meme online market through their advertising.

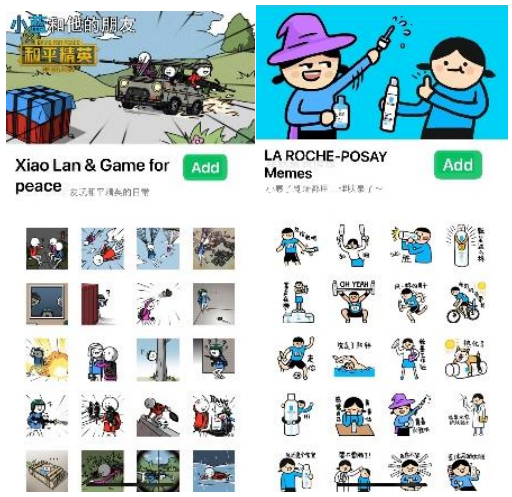

Figure6: LA ROCHE-POSAY and Game for peace's brand promotion with XiaoLan.

Using Meme for brand marketing can be very useful for four main reasons:

1 - Memes fit with social media communication. They are fun and can help form interactive circles among social media followers, which creates a sense of belonging. This is because they have the same identity and interests.

2 - Meme-based content is seditious. Based on the meaning of meme, it can be shared very easily. Therefore, the content of a meme is generally re-posted by other Internet users. When the usage of a meme starts, brands can easily spread their advertisements. 
3 - Meme production costs are low. Screenshots from movies, photographs, or online images can be the material for a meme. People do not need to take original videos or photos themselves.

4 - Memes can be adapted to suit brand tonality. The distraction of existing media can be eliminated by adding new titles or fonts. In addition, memes have a DIY element and low-resolution content is considered acceptable, so there is no need to worry if design skills are limited are lacking entirely.

According to research, in order to conduct successful brand marketing, in addition to timely grasping relevant hot spots and combining them, memes must remain consistent with the brand. Memes often use sarcasm, social commentary, or nostalgia, meaning that high-quality memes can be fun with their own insights. However, as memes can also be rude or offensive, marketers must be careful not to annoy fans during the course of their meme marketing.

The key to meme marketing is to remain authentic and not be overly conservative. Avant-garde content can demonstrate that a brand has personality and a unique voice, which can make brand differentiation in similar products, appeal to young customer groups, and increase popularity. A short explosion can have a great impact.

\section{CONCLUSION}

In the image consumption, the appearance of meme has become an unprecedented carnival form of social entertainment. The emphasis is no longer on the medium or the message, different to Marshall McLuhan (1964). Instead, the emphasis is on creating and exploring new archives of visual materials, mapping them to discover connections between what is visual and the culture as a whole, and realizing that what we are learning to see above all is change on the global scale (Nicholas Mirzoeff,2015) ${ }^{i x}$ [9]. Human image wars and image consumption have a long history and keep changing, from religious paintings, advertisement photographs. Everyone may now engage in meme culture, The visualization of memes can alleviate social anxiety and resolve social awkwardness. Memes can also restore social scene, reproduce social modality, make up the online drawback of the communication which can't really face to face, for the brand, the expression of emerging marketing, can let the brand get rid of strange figure, more integrated into the consumer groups. Strong meme interaction between a brand and users can enhance users' sense of participation and identity, which can develop them into loyal users of that brand.

\section{REFERENCES}

[1] Jessica Evans and Stuart Hall (eds) (1999). Visual Culture: The Reader. SAGE

[2] W.J.T.Mitchell, picture Theory: Essays on verbal and visual representation, University of Chicago Press, Chicago, 1995, p.11

[3] Stuart Hall, Representation : cultural representations and signifying practices. The open university, 1997, Introduction

[4] Kevin Michael DeLuca; Image politics: New Rhetoric of Environmental Activism; Taylor \& Francis Inc, 2005

[5] Gotthold Ephraim Lessing (1729-1781), Laocoon; An Essay on the Limits of Painting and Poetry (1766). P1

[6] Evans, Jessica \& Hall, Professor. (1999), Zooming In: Histories of Photography in China by Wu Hung,. Visual Culture: The Reader p192

[7] Evans, Jessica \& Hall, Professor. (1999), Zooming In: Histories of Photography in China by Wu Hung,. Visual Culture: The Reader p218

[8] Evans, Jessica \& Hall, Professor. (1999). Visual Culture: The Reader

[9] Mirzoeff, N. .(2015). How to see the world. P56. 